\title{
Achieving Weight Coverage for an Autonomous Driving System with Search-based Test Generation (HOP Track at GECCO 2021)
}

\author{
Thomas Laurent \\ Lero \& University College Dublin \\ Ireland \\ thomas.laurent@ucdconnect.ie \\ Fuyuki Ishikawa \\ National Institute of Informatics \\ Tokyo, Japan \\ f-ishikawa@nii.ac.jp
}

\author{
Paolo Arcaini \\ National Institute of Informatics \\ Tokyo, Japan \\ arcaini@nii.ac.jp \\ Anthony Ventresque \\ Lero \& University College Dublin \\ Ireland \\ anthony.ventresque@ucd.ie
}

\begin{abstract}
Autonomous Driving Systems (ADSs) are complex critical systems that must be thoroughly tested. Still, assessing the strength of tests for ADSs is an open and complex problem. Weight Coverage is a test criterion targeting ADSs which are based on a weighted cost function. It measures how much each weight, related to different aspects of the ADS's decision process, is involved in the decisions taken in a test scenario. All weights/aspects should be involved for a strong test suite. Although weight coverage can measure the quality of a test suite, it does not provide clear guidance for test generation. This work proposes weight coverage-driven search-based test generation for ADSs. It describes and compares three designs of the search process: a single-objective search aiming at generating a test covering a single weight; a multi-objective search where each objective targets a different weight; and a single-objective search where the fitness function is an aggregate function representing the coverage over multiple weights. Experiments using an ADS system provided by our industry partner show the validity of the method and provide insights into the benefits of each search design.

This Hot-off-the-Press paper summarises the paper [2]: T. Laurent, P. Arcaini, F. Ishikawa and A. Ventresque, "Achieving Weight Coverage for an Autonomous Driving System with Search-based Test Generation", 25th International Conference on Engineering of Complex Computer Systems (ICECCS 2020).
\end{abstract}

\section{CCS CONCEPTS}

- Computer systems organization $\rightarrow$ Embedded and cyberphysical systems; • Software and its engineering $\rightarrow$ Searchbased software engineering.

\section{KEYWORDS}

search-based testing, autonomous driving, weight coverage

\footnotetext{
Permission to make digital or hard copies of part or all of this work for personal or classroom use is granted without fee provided that copies are not made or distributed for profit or commercial advantage and that copies bear this notice and the full citation on the first page. Copyrights for third-party components of this work must be honored. For all other uses, contact the owner/author(s)

GECCO '21 Companion, fuly 10-14, 2021, Lille, France

(c) 2021 Copyright held by the owner/author(s).

ACM ISBN 978-1-4503-8351-6/21/07.

https://doi.org/10.1145/3449726.3462723
}

ACM Reference Format:

Thomas Laurent, Paolo Arcaini, Fuyuki Ishikawa, and Anthony Ventresque. 2021. Achieving Weight Coverage for an Autonomous Driving System with Search-based Test Generation (HOP Track at GECCO 2021). In 2021 Genetic and Evolutionary Computation Conference Companion (GECCO '21 Companion), July 10-14, 2021, Lille, France. ACM, New York, NY, USA, 2 pages. https://doi.org/10.1145/3449726.3462723

\section{BACKGROUND AND PROPOSED METHOD}

Autonomous Driving Systems (ADSs) represent a complex and active area of research, which promises to have a large impact on society. As such, they have recently been the focus of a lot of work. As they are eventually deployed on the streets, they must be thoroughly tested in order to avoid possibly fatal consequences.

Assessing whether a set of driving scenarios tests the ADS thoroughly enough, or if more testing is needed, is an open problem. To address this problem, some coverage criteria have been defined, such as identifying diverse scenario types to include in the test suite, or evaluating scenarios that trigger all the possible "types of decisions" of the ADS, as required by weight coverage [1]. This work focuses on the use of weight coverage to drive the testing effort.

Weight coverage is computed using a mutation-based approach. It creates several mutated ADSs having a different value than the original system for one of the weights. The approach then runs the original and mutant ADS on the test scenarios and compares the results. If the mutated ADS produces a different result, then the weight is covered. The smaller the difference between the mutated and original value of the weight, i.e., the mutation level, the better the coverage. Comparison of the results is performed using a delta function $\Delta$. Three delta functions can be used: $\Delta^{P}$ that compares the paths produced by both systems, $\Delta^{S}$ that compares the safety of the paths, and $\Delta^{C}$ that compares the comfort of the paths.

Although weight coverage assesses how well a set of scenarios tests the ADS, it does not give clues towards the missing scenarios to increase coverage. If a weight is not (or badly) covered, it is unclear what scenario would cover it. Thus, the testing process remains based on manual and costly exploration, hence the need for automated test generation.

This work proposes weight coverage-driven search-based generation for ADS. In the search individuals represent scenarios. In particular, we define a baseline scenario $S_{\bar{v}}$ in which some fields $\bar{v}$ 
(e.g., initial position, speed, direction, acceleration of a given scenario car) are not specified: these are the search variables. Given some values $\bar{v}^{\prime}$ for the searched variables, we will indicate with $S_{\bar{v}^{\prime}}$ the scenario $S_{\bar{v}}$ with the new values $\bar{v}^{\prime}$.

This work describes and compares three designs of the search process, SWA, MWAMO, and MWAso, detailed as follows.

Single-weight approach (SWA) In this variant of the approach, given a weight $w_{i}$, we search for a scenario that achieves weight coverage for $w_{i}$. The search is a single-objective search and the fitness function is simply the coverage achieved by a scenario, i.e. scenarios detecting small levels of mutation for weight $w_{i}$ have better fitness. The single weight approach should be run for each weight in the system in order to produce a test suite with full coverage, and would produce one scenario for each weight. As a single scenario could cover multiple weights, this work also explores approaches targeting multiple weights. Although computing the fitness in these approaches is more expensive (weight coverage must be computed for multiple weights instead of one), they should produce smaller test suites with less specialised scenarios.

Multi-weight multi-objective approach $\left(M_{W} A_{M O}\right)$ In this variant, given $n$ weights $w_{1}, \ldots, w_{n}$ that we want to cover, we search for a scenario that achieves weight coverage on all weights. The search is a multi-objective search with $n$ fitness functions, each function fit $_{i}$ being the coverage achieved on weight $w_{i}$.

Multi-weight single-objective approach (MWAso) In this variant, like in MWAMO, given $n$ weights $w_{1}, \ldots, w_{n}$ that we want to cover, we search for a scenario that achieves weight coverage on all weights. Similarly to SWA, the search is single-objective, but the fitness function aggregates the coverage achieved on the different weights. The aggregation function is designed so that a scenario covering more weights is always preferred to one covering fewer weights, and a scenario covering one of the weights with a smaller mutation level is preferred if it covers the other weights as well.

\section{SUMMARY OF THE RESULTS}

All three methods were applied to an ADS provided by our industry partner. The experiments targeted 4 of the system's weights, and used 3 search spaces (i.e., baseline scenarios and field intervals). All experiments were repeated 5 times. This section presents the main results from these experiments, first on the performance of each method, and then on the comparison between methods.

\subsection{Coverage of each method}

Single-weight approach (SWA) Results show that this method reliably finds scenarios achieving coverage, often detecting the smallest mutation level for the targeted weight. They also show that, although the scenarios the method produces are specialised to cover the target weight, they can also cover other weights, although most scenarios only detect higher levels of mutation for non-target weights. This shows that scenarios can cover multiple weights, although SWA does not find scenarios both covering multiple weights and detecting small mutation levels for all weights, further motivating the inquiry into the two multi-weight approaches.

Multi-weight multi-objective approach (MWAMO) Results show that this method finds scenarios that cover the 4 weights considered during the search, often detecting small mutation levels. It can thus produce small test suites achieving good coverage.

Multi-weight single-objective approach $\left(M W A_{S O}\right)$ Results show that this method finds scenarios that cover the 4 weights considered during the search, although the mutation levels detected are higher than with scenarios produced by MWAMO.

\subsection{Comparison between the three approaches}

The non-parametric Wilcoxon test and the $\hat{A}_{12}$ statistic provided a way to compare the approaches. These tests let us compare the overall coverage achieved by the set of 4 scenarios produced by SWA, each targeting one of the weights used in the experiments, with the best scenario (covering the most weights and detecting the lowest mutation levels) produced by each of MWAMO and MWAso.

These results show that SWA performs best out of the three methods in terms of coverage, which is expected as it produces four scenarios while the other approaches produce one. Still, as mentioned above, $M_{W} A_{M O}$ and MWAso produce scenarios covering all weights, leading to smaller test suite size. Privileging test suite size over optimal coverage could be desirable. Of the two multi-weight approaches, MWA $A_{M O}$ systematically performs better, thus the "true" multi-objective approach should be preferred over the combined objectives approach. Interestingly, when considering the comfort coverage objective $\left(\Delta^{C}\right)$, a much harder problem, all three approaches perform similarly, as the main issue becomes finding a covering scenario, rather than a scenario detecting smaller levels of mutation.

\section{CONCLUSIONS}

The main theoretical challenge of this work was to obtain scalability. To this aim, in the fitness function (which is time costly, being based on simulation), the mutants are evaluated in a given order. In the same spirit, in the design of the fitness function of MWAso, we prioritised the different levels of coverage of the different weights. We believe that future works on search-based testing for ADSs should tackle the problem of scalability as main challenge.

\section{ACKNOWLEDGMENTS}

This work was supported supported, in part, by Science Foundation Ireland grant 13/RC/2094_P2. T. Laurent is supported by an Irish Research Council grant (GOIPG/2017/1829). P. Arcaini and F. Ishikawa are supported by ERATO HASUO Metamathematics for Systems Design Project (No. JPMJER1603), JST. We thank our industry partner Mazda for providing the software used as target in our work and discussing principles in testing and improving realworld automotive systems: the software is a prototype constructed for the purpose of evaluating new testing methods, and its quality has no relation with the quality of Mazda products.

\section{REFERENCES}

[1] Thomas Laurent, Paolo Arcaini, Fuyuki Ishikawa, and Anthony Ventresque. 2019. A Mutation-based Approach for Assessing Weight Coverage of a Path Planner. In 26th Asia-Pacific Software Engineering Conference (APSEC). 94-101. https: //doi.org/10.1109/APSEC48747.2019.00022

[2] Thomas Laurent, Paolo Arcaini, Fuyuki Ishikawa, and Anthony Ventresque. 2020. Achieving Weight Coverage for an Autonomous Driving System with Search-based Test Generation. In 2020 25th International Conference on Engineering of Complex Computer Systems (ICECCS). 93-102. https://doi.org/10.1109/ICECCS51672.2020. 00018 